Volume 70, Number 2, Pages 731-743(2021)

DOI: $10.31801 /$ cfsuasmas. 865647

ISSN 1303-5991 E-ISSN 2618-6470

Received by the editors: January 20, 2021; Accepted: April 7, 2021

\title{
NUMERICAL SOLUTION TO AN INTEGRAL EQUATION FOR THE KTH MOMENT FUNCTION OF A GEOMETRIC PROCESS
}

\author{
Mustafa Hilmi PEKALP ${ }^{1}$ and Ayşenur AYDOĞDU ${ }^{2}$ \\ ${ }^{1}$ Ankara University, Faculty of Applied Sciences, Department of Actuarial Sciences, \\ Ankara, TURKEY \\ ${ }^{2}$ Ankara University, Faculty of Science, Department of Mathematics, Ankara, TURKEY
}

\begin{abstract}
In this paper, an integral equation for the kth moment function of a geometric process is derived as a generalization of the lower-order moments of the process. We propose a general solution to solve this integral equation by using the numerical method, namely trapezoidal integration rule. The general solution is reduced to the numerical solution of the integral equations which will be given for the third and fourth moment functions to compute the skewness and kurtosis of a geometric process. To illustrate the numerical method, we assume gamma, Weibull and lognormal distributions for the first interarrival time of the geometric process.
\end{abstract}

\section{INTRODUCTION}

The geometric process (GP), which is a natural generalization of a renewal process (RP), is an important stochastic monotone model used in many areas of statistics and applied probability, especially for statistical analysis of series of events. Since its introduction by [9], many researcher and authors made a significance effort on GP by publishing more than 200 papers. For instance, the GP has been used as a model in modelling of an epidemic disease [8], software reliability [16, 18, maintenance [23, warranty analysis [5, 12$]$ and electricity prices $[7]$. This process is defined as follows.

Definition 1. Let $\{N(t), t \geq 0\}$ be a counting process (CP) and $X_{i}$ be the interarrival time between $(i-1)$ th and ith event of this process for $i=1,2, \ldots$ The $C P$ $\{N(t), t \geq 0\}$ is called a GP with the ratio parameter a if there exists a real number $a>0$ such that $a^{i-1} X_{i}, i=1,2, \ldots$ are independent and identically distributed

2020 Mathematics Subject Classification. Primary 60K99, 65R20; Secondary 60E10 ,62E10. Keywords and phrases. Geometric process, moment functions, skewness and kurtosis, numerical solution.

mpekalp@ankara.edu.tr-Corresponding author; aysenurydgd@gmail.com

(D) 0000-0002-5183-8394; 0000-0001-6439-8431.

(C)2021 Ankara University Communications Faculty of Sciences University of Ankara-Series A1 Mathematics and Statistics 
(iid) random variables with a distribution function $F$, where $F$ is the distribution function of the first interarrival time $X_{1}$.

The GP is also called quasi-renewal process with ratio parameter $\alpha=1 / a$ by 18 .

Let $\{N(t), t \geq 0\}$ be a GP with the ratio parameter $a$ and $F_{i}$ be the distribution function of $X_{i}$ for $i=1,2, \ldots$ Then, it is easy to verify that $F_{i}(x)=F\left(a^{i-1} x\right)$ for $i=1,2, \ldots$. Further, it can be shown that GP is stochastically increasing if $a<1$ and stochastically decreasing if $a>1$. When $a=1$, GP reduces to a RP.

By considering the distribution functions of the interarrival times, one of the important differences between RP and GP can be given as follows. In RP, the distribution function of the interarrival times remains same over $i$ 's, that is $F_{i}(x)=$ $F(x)$ for $i=1,2, \ldots$ However, in GP, the distribution of the interarrival time $X_{i}$ does not remain same over $i$ 's, that is $F_{i}(x)=F\left(a^{i-1} x\right)$ for $i=1,2, \ldots$ This provides some advantages to the GP in applications, in particular for reliability mathematics since it can be used as a model for deteriorating systems which may have decreasing working times between failures. In order to understand the place of the GP model in the literature, see the following papers, [1, 19, 20, 21,22].

Let $\{N(t), t \geq 0\}$ be a GP with ratio parameter $a$. Set $S_{0}=0$ and $S_{n}=$ $\sum_{i=1}^{n} X_{i}$ for $n=1,2, \ldots$ Thus, $S_{n}$ is called $n$th arrival time of the GP. The distribution function of $S_{n}$ is given by $F_{1} * F_{2} * \cdots * F_{n}(t)$, where $*$ denotes the Stieltjes convolution and $F_{i}(t)$ for $i=1,2, \ldots$ is the distribution function of $X_{i}$. Further, since the events $\left(S_{n} \leq t\right)$ and $(N(t) \geq n)$ are equivalent, the probability distribution of the random variable $N(t)$ is given by

$$
P(N(t)=n)=F_{1} * \cdots * F_{n}(t)-F_{1} * \cdots * F_{n+1}(t)
$$

for each fixed $t \geq 0$.

Now, let give the following theorem which states the existence of the moments of the GP. The proof of this theorem can be found in many manuscripts and monographs, for example, 6, 11.

Theorem 2. Consider the GP $\{N(t), t \geq 0\}$ with ratio parameter a. If $a \leq 1$, then $M_{k}(t)=E\left(N^{k}(t)\right)<\infty$ for all $t \geq 0$ and $k \geq 0$. If $a>1$ and $F(t)>0$ for all $t>0$, then the moments of $N(t)$ are infinite.

Let $\{N(t), t \geq 0\}$ be a GP with ratio parameter $a$. The mean value function of a GP, which is also called the geometric function, is given by $M_{1}(t)=E(N(t))$. $M_{1}(t)$ is the number of the events that have occurred by time $t$. The geometric function $M_{1}(t)$ satisfies the following integral equation.

$$
M_{1}(t)=F(t)+\int_{0}^{t} M_{1}(a(t-x)) d F(x), t \geq 0 .
$$

The second moment function of a GP is given by $M_{2}(t)=E\left(N^{2}(t)\right)$. 15 show that $M_{2}(t)$ satisfies the following integral equation.

$$
M_{2}(t)=2 M_{1}(t)-F(t)+\int_{0}^{t} M_{2}(a(t-x)) d F(x), t \geq 0 .
$$


According to the Theorem 1, for $a \leq 1$, the geometric function $M_{1}(t)$ and the second moment function $M_{2}(t)$ are finite for all $t \geq 0$. Furthermore, if $F$ is continuous, then the integral equations (1) and (2) can be solved uniquely although $M_{1}(t)$ and $M_{2}(t)$ cannot be obtained in analytical forms. In the case of $a>1$, $M_{1}(t)$ and $M_{2}(t)$ are infinite for all $t>0$.

When the GP model is used as a model for the data sets come from series of event, the distribution function of the first interarrival time is assumed to be one of four common lifetime distributions as exponential, gamma, Weibull and lognormal. See 13 for the details how to discriminate the lifetime distributions in GP model. Under a lifetime distribution assumption, it is of importance to calculate the moment functions of the GP. Many researchers and authors made some studies on the first and second moment functions of the GP by considering these lifetime distributions. [6] deal with the boundary problem for $M_{1}(t)$. 17] propose a numerical method, namely trapezoidal integration rule, for $M_{1}(t)$ with the help of the integral equation (1). In addition, [4] and [3] suggest power series expansions for $M_{1}(t)$ depending on the integral equation (1). 2 obtain the numerical calculation and Monte Carlo estimation of the variance function, which is $M_{2}(t)-M_{1}^{2}(t)$, by using the convolution of the distribution functions. Alternative methods for computing $M_{2}(t)$ are given in 15 . They adapt the Tang and Lam's method to $M_{2}(t)$ and propose a power series expansion for $M_{2}(t)$ with the help of the integral equation (2). Further, 14 show the asymptotic solution of the integral equation for the second moment function. To the best of our knowledge, in the literature, there is no study about the higher-order moment functions of the GP. However, in

order to calculate, for instance, the skewness and kurtosis of the process, the third and fourth moment functions are required. Moreover, to compare the estimators proposed for some parameters of GP and to examine some theoretical properties of the process, higher-order moment functions should be known.

The rest of the paper organized as follows. In section 2, firstly, we obtain the integral equations for the third and fourth moment functions of the GP. Then, a generalization of the integral equation for kth moment function of the GP is given. We adapt the Tang and Lam's numerical method for the kth moment function of the GP with the help of the integral equation given for this function. Then, we reduce this general approximation to third and fourth moment functions of the GP in Section 3. Illustrative examples are provided in Section 4. Conclusion remarks are presented in Section 5.

\section{INTEGRAL EQUATIONS FOR THE MOMENT FUNCTIONS OF THE GP}

In this section, firstly, integral equations for the third and fourth moment functions of the GP are obtained. Then, in general, we present an integral equation for the kth moment function of the GP. 
Let $\{N(t), t \geq 0\}$ be a GP with ratio parameter $a$ and let us assume that the first interarrival time $X_{1}$ follows the distribution function $F$. The third moment function of the GP is given by $M_{3}(t)=E\left(N^{3}(t)\right), t \geq 0$. Conditioning on the first interarrival time $X_{1}$, we have

$$
M_{3}(t)=E\left(N^{3}(t)\right)=\int_{0}^{\infty} E\left(N^{3}(t) \mid X_{1}=x\right) d F(x) .
$$

Since $E\left(N^{3}(t) \mid X_{1}=x\right)=E(1+N(a(t-x)))^{3}, x<t$ and $E\left(N^{3}(t) \mid X_{1}=x\right)=$ $0, x \geq t$, we rewrite the equality as follows.

$$
\begin{aligned}
M_{3}(t) & =\int_{0}^{t} E(1+N(a(t-x)))^{3} d F(x) \\
& =\int_{0}^{t} d F(x)+3 \int_{0}^{t} E(N(a(t-x))) d F(x) \\
& +3 \int_{0}^{t} E\left(N^{2}(a(t-x))\right) d F(x)+\int_{0}^{t} E\left(N^{3}(a(t-x))\right) d F(x) \\
& =F(t)+3 \int_{0}^{t} M_{1}(a(t-x)) d F(x)+3 \int_{0}^{t} M_{2}(a(t-x)) d F(x) \\
& +\int_{0}^{t} M_{3}(a(t-x)) d F(x) .
\end{aligned}
$$

Using the integral equations given in (1) and (2), we have

$$
\begin{aligned}
M_{3}(t) & =F(t)+3\left(M_{1}(t)-F(t)\right)+3\left(M_{2}(t)-2 M_{1}(t)+F(t)\right) \\
& +\int_{0}^{t} M_{3}(a(t-x)) d F(x) .
\end{aligned}
$$

Simplifying the expression, we obtain

$$
M_{3}(t)=3 M_{2}(t)-3 M_{1}(t)+F(t)+\int_{0}^{t} M_{3}(a(t-x)) d F(x), t \geq 0 .
$$

The fourth moment function of the GP is defined by $M_{4}(t)=E\left(N^{4}(t)\right), t \geq 0$. Using similar arguments in the derivation of the integral equation for $M_{3}(t)$, we have

$$
\begin{aligned}
& M_{4}(t)=4 M_{3}(t)-6 M_{2}(t)+4 M_{1}(t)-F(t) \\
& +\int_{0}^{t} M_{4}(a(t-x)) d F(x), t \geq 0
\end{aligned}
$$

Following theorem states the kth moment function of a GP model. 
Theorem 3. For any $k \in \mathbb{N}$, the kth moment function $M_{k}(t)$ of the GP is given by

$$
M_{k}(t)=\sum_{j=0}^{k-1}(-1)^{k-1-j}\left(\begin{array}{c}
k \\
j
\end{array}\right) M_{j}(t)+\int_{0}^{t} M_{k}(a(t-x)) d F(x), t \geq 0,
$$

where $M_{0}(t)=F(t)$.

Proof. The proof of (5) is given by using the mathematical induction. It is obvious that (5) holds for $k=1$ when we consider equation (1). Now, let us assume that (5) holds for any integer $k$ and show that it also holds for integer $k+1$. The $(k+1)$ th moment function of the GP is defined by $M_{k+1}(t)=E\left(N^{k+1}(t)\right), t \geq 0$. Conditioning on the first interarrival time $X_{1}$, we have

$$
\begin{aligned}
M_{k+1}(t) & =\int_{0}^{t} E(1+N(a(t-x)))^{k+1} d F(x) \\
& =\sum_{j=0}^{k+1}\left(\begin{array}{c}
k+1 \\
j
\end{array}\right) \int_{0}^{t} M_{j}(a(t-x)) d F(x) .
\end{aligned}
$$

The following equation can be written by separating the $(k+1)$ th term.

$$
M_{k+1}(t)=\sum_{j=0}^{k}\left(\begin{array}{c}
k+1 \\
j
\end{array}\right) \int_{0}^{t} M_{j}(a(t-x)) d F(x)+\int_{0}^{t} M_{k+1}(a(t-x)) d F(x) .
$$

Since we assume that (5) holds for any integer $k$, we have

$$
\begin{aligned}
M_{k+1}(t) & =\sum_{j=0}^{k}\left(\begin{array}{c}
k+1 \\
j
\end{array}\right)\left(M_{j}(t)-\sum_{i=0}^{j-1}(-1)^{j-1-i}\left(\begin{array}{l}
j \\
i
\end{array}\right) M_{i}(t)\right) \\
& +\int_{0}^{t} M_{k+1}(a(t-x)) d F(x) \\
& =\sum_{j=0}^{k}\left(\begin{array}{c}
k+1 \\
j
\end{array}\right)\left(\sum_{i=0}^{j}(-1)^{j-i}\left(\begin{array}{l}
j \\
i
\end{array}\right) M_{i}(t)\right) \\
& +\int_{0}^{t} M_{k+1}(a(t-x)) d F(x) .
\end{aligned}
$$

When we rearrange the terms, we obtain

$$
M_{k+1}(t)=\sum_{i=0}^{k}\left(\sum_{j=i}^{k}(-1)^{j-i}\left(\begin{array}{c}
k+1 \\
j
\end{array}\right)\left(\begin{array}{l}
j \\
i
\end{array}\right)\right) M_{i}(t)+\int_{0}^{t} M_{k+1}(a(t-x)) d F(x) .
$$


Since the identities $\left(\begin{array}{c}k+1 \\ j\end{array}\right)\left(\begin{array}{l}j \\ i\end{array}\right)=\left(\begin{array}{c}k+1 \\ i\end{array}\right)\left(\begin{array}{c}k+1-i \\ j-i\end{array}\right)$ and $\sum_{j=i}^{k}(-1)^{j-i}\left(\begin{array}{c}k+1-i \\ j-i\end{array}\right)=$ $(-1)^{k-i}$ hold,

$$
M_{k+1}(t)=\sum_{i=0}^{k}(-1)^{k-i}\left(\begin{array}{c}
k+1 \\
i
\end{array}\right) M_{i}(t)+\int_{0}^{t} M_{k+1}(a(t-x)) d F(x) .
$$

Hence, the proof is completed.

According to the Theorem 1, for $a \leq 1$, the function $M_{k}(t)$ is finite for all $t \geq 0$. Furthermore, if $F$ is continuous, then the integral equation (5) can be solved uniquely although this function does not have an analytical form. We discuss this problem in the next section. In the case of $a>1, M_{k}(t)$ is infinite for all $t>0$.

\section{NUMERICAL SOLUTION}

In this section, we give a method based on the trapezoidal integration rule for the numerical solution of the integral equation (5). This solution is obtained by recursive calculations with respect to $k$. Now, let us remind the trapezoidal integration rule as follows.

According to the trapezoidal integration rule, an integral $\int_{a}^{b} g(t) d t$ can be calculated numerically as

$$
\int_{a}^{b} g(t) d t \approx \sum_{i=1}^{n} \frac{g\left(t_{i-1}\right)+g\left(t_{i}\right)}{2}\left(t_{i}-t_{i-1}\right)=\frac{h}{2} g\left(t_{0}\right)+h \sum_{i=1}^{n-1} g\left(t_{i}\right)+\frac{h}{2} g\left(t_{n}\right),
$$

where $\left\{t_{0}, t_{1}, \ldots, t_{n}\right\}$ is a partition of the interval $[a, b]$ such that $a=t_{0}<t_{1}<$ $\cdots<t_{n}=b, t_{i}=a+i h, i=0,1, \ldots, n$ and $h=\frac{b-a}{n}$. The approximation gives more precise results as the number of the partition increases.

Let $\{N(t), t \geq 0\}$ be a GP with ratio parameter $a<1$. Assume that the first interarrival time $X_{1}$ has probability density function $f$. Then, the integral equation (5) can be written as

$$
M_{k}(t)=\sum_{j=0}^{k-1}(-1)^{k-1-j}\left(\begin{array}{l}
k \\
j
\end{array}\right) M_{j}(t)+\int_{0}^{t} M_{k}(a(t-x)) f(x) d x, t \geq 0 .
$$

If we substitute $s=a(t-x)$, we have

$$
M_{k}(t)=\sum_{j=0}^{k-1}(-1)^{k-1-j}\left(\begin{array}{c}
k \\
j
\end{array}\right) M_{j}(t)+\frac{1}{a} \int_{0}^{a t} M_{k}(s) f\left(t-\frac{s}{a}\right) d s .
$$

Assume that $T>0, t \in[0, T]$ and $f(0)=0$. Let $\left\{t_{0}, t_{1}, \ldots, t_{n}\right\}$ be a partition of the interval $[0, T]$ such that $0=t_{0}<t_{1}<\cdots<t_{n}=T$. Take the step width $h=\frac{T}{n}$ and $t_{i}=i h$ for $i=0,1, \ldots, n$. By (7), we have 


$$
\begin{aligned}
M_{k}\left(t_{i}\right) & =\sum_{j=0}^{k-1}(-1)^{k-1-j}\left(\begin{array}{c}
k \\
j
\end{array}\right) M_{j}\left(t_{i}\right) \\
& +\frac{1}{a} \int_{0}^{t_{\lfloor a i\rfloor}} M_{k}(s) f\left(t_{i}-\frac{s}{a}\right) d s \\
& +\frac{1}{a} \int_{t_{\lfloor a i\rfloor}}^{a t_{i}} M_{k}(s) f\left(t_{i}-\frac{s}{a}\right) d s .
\end{aligned}
$$

where $\lfloor$.$\rfloor is the greatest integer function. Since a t_{i}$ does not have to belong to this partition, the interval $\left[0, a t_{i}\right]$ is divided into two subsets as $\left[0, t_{\lfloor a i\rfloor}\right]$ and $\left[t_{\lfloor a i\rfloor}, a t_{i}\right]$. Now, let us define $I_{1}$ and $I_{2}$ as

$$
I_{1}=\frac{1}{a} \int_{0}^{t_{\lfloor a i\rfloor}} M_{k}(s) f\left(t_{i}-\frac{s}{a}\right) d s
$$

and

$$
I_{2}=\frac{1}{a} \int_{t_{\lfloor a i\rfloor}}^{a t_{i}} M_{k}(s) f\left(t_{i}-\frac{s}{a}\right) d s
$$

Considering the trapezoidal integration rule with the partition $\{0, h, 2 h, \ldots,[a i] h\}$ of the interval $\left[0, t_{\lfloor a i\rfloor}\right]$, we obtain

$$
\begin{aligned}
I_{1} & =\frac{1}{a} \int_{0}^{t_{\lfloor a i\rfloor}} M_{k}(s) f\left(t_{i}-\frac{s}{a}\right) d s \\
& \approx \frac{h}{2 a} M_{k}\left(t_{0}\right) f\left(t_{i}-\frac{t_{0}}{a}\right)+\frac{h}{a} \sum_{j=1}^{\lfloor a i\rfloor-1} M_{k}\left(t_{j}\right) f\left(t_{i}-\frac{t_{j}}{a}\right) \\
& +\frac{h}{2 a} M_{k}\left(t_{\lfloor a i\rfloor}\right) f\left(t_{i}-\frac{t_{\lfloor a i\rfloor}}{a}\right) \\
& =\frac{h}{a} \sum_{j=1}^{\lfloor a i\rfloor-1} M_{k}\left(t_{j}\right) f\left(t_{i}-\frac{t_{j}}{a}\right) \\
& +\frac{h}{2 a} M_{k}\left(t_{\lfloor a i\rfloor}\right) f\left(t_{i}-\frac{t_{\lfloor a i\rfloor}}{a}\right),
\end{aligned}
$$

where $M_{k}(0)=0$. To calculate the integral $I_{2}$, it can be again used the trapezoidal integration rule with two points (the values of the lower and upper bounds) on the interval $\left[t_{\lfloor a i\rfloor}, a t_{i}\right]$. Hence,

$$
\begin{aligned}
I_{2} & =\frac{1}{a} \int_{t_{\lfloor a i\rfloor}}^{a t_{i}} M_{k}(s) f\left(t_{i}-\frac{s}{a}\right) d s \\
& \approx \frac{a t_{i}-t_{\lfloor a i\rfloor}}{2 a}\left(M_{k}\left(t_{\lfloor a i\rfloor}\right) f\left(t_{i}-\frac{t_{\lfloor a i\rfloor}}{a}\right)+M_{k}\left(a t_{i}\right) f(0)\right)
\end{aligned}
$$




$$
=\frac{a t_{i}-t_{\lfloor a i\rfloor}}{2 a}\left(M_{k}\left(t_{\lfloor a i\rfloor}\right) f\left(t_{i}-\frac{t_{\lfloor a i\rfloor}}{a}\right)\right) .
$$

By using the equations (9) and (10) into the equation (8), we have

$$
\begin{aligned}
M_{k}\left(t_{i}\right) & \approx \sum_{j=0}^{k-1}(-1)^{k-1-j}\left(\begin{array}{c}
k \\
j
\end{array}\right) M_{j}\left(t_{i}\right) \\
& +\frac{h}{a} \sum_{j=1}^{\lfloor a i\rfloor-1} M_{k}\left(t_{j}\right) f\left(t_{i}-\frac{t_{j}}{a}\right) \\
& +\frac{h}{2 a} M_{k}\left(t_{\lfloor a i\rfloor}\right) f\left(t_{i}-\frac{t_{\lfloor a i\rfloor}}{a}\right) \\
& +\frac{a t_{i}-t_{\lfloor a i\rfloor}}{2 a}\left(M_{k}\left(t_{\lfloor a i\rfloor}\right) f\left(t_{i}-\frac{t_{\lfloor a i\rfloor}}{a}\right)\right) .
\end{aligned}
$$

Denote the approximate value of $M_{k}\left(t_{i}\right)$ as $\tilde{M}_{k}\left(t_{i}\right)$. Then, for any given $k \geq 1$ and $i=0,1, \ldots, n$, the values of $\tilde{M}_{k}\left(t_{i}\right)$ can be recursively calculated as

$$
\begin{aligned}
\tilde{M}_{k}\left(t_{i}\right) & =\sum_{j=0}^{k-1}(-1)^{k-1-j}\left(\begin{array}{c}
k \\
j
\end{array}\right) \tilde{M}_{j}\left(t_{i}\right) \\
& +\frac{h}{a} \sum_{j=1}^{\lfloor a i\rfloor-1} \tilde{M}_{k}\left(t_{j}\right) f\left(t_{i}-\frac{t_{j}}{a}\right) \\
& +\frac{h}{2 a} \tilde{M}_{k}\left(t_{\lfloor a i\rfloor}\right) f\left(t_{i}-\frac{t_{\lfloor a i\rfloor}}{a}\right) \\
& +\frac{a t_{i}-t_{\lfloor a i\rfloor}}{2 a}\left(\tilde{M}_{k}\left(t_{\lfloor a i\rfloor}\right) f\left(t_{i}-\frac{t_{\lfloor a i\rfloor}}{a}\right)\right),
\end{aligned}
$$

where $\tilde{M}_{k}(0)=M_{k}(0)=0$.

Let us reduce this general solution to the numerical solution of integral equations (3) and (4). By (11), the values of $\tilde{M}_{3}\left(t_{i}\right)$ and $\tilde{M}_{4}\left(t_{i}\right)$ can be calculated as

$$
\begin{aligned}
\tilde{M}_{3}\left(t_{i}\right) & =3 \tilde{M}_{2}\left(t_{i}\right)-3 \tilde{M}_{1}\left(t_{i}\right)+F\left(t_{i}\right) \\
& +\frac{h}{a} \sum_{j=1}^{\lfloor a i\rfloor-1} \tilde{M}_{3}\left(t_{j}\right) f\left(t_{i}-\frac{t_{j}}{a}\right) \\
& +\frac{h}{2 a} \tilde{M}_{3}\left(t_{\lfloor a i\rfloor}\right) f\left(t_{i}-\frac{t_{\lfloor a i\rfloor}}{a}\right) \\
& +\frac{a t_{i}-t_{\lfloor a i\rfloor}}{2 a}\left(\tilde{M}_{3}\left(t_{\lfloor a i\rfloor}\right) f\left(t_{i}-\frac{t_{\lfloor a i\rfloor}}{a}\right)\right)
\end{aligned}
$$


and

$$
\begin{aligned}
\tilde{M}_{4}\left(t_{i}\right) & =4 \tilde{M}_{3}\left(t_{i}\right)-6 \tilde{M}_{2}\left(t_{i}\right)+4 \tilde{M}_{1}\left(t_{i}\right)-F\left(t_{i}\right) \\
& +\frac{h}{a} \sum_{j=1}^{\lfloor a i\rfloor-1} \tilde{M}_{4}\left(t_{j}\right) f\left(t_{i}-\frac{t_{j}}{a}\right) \\
& +\frac{h}{2 a} \tilde{M}_{4}\left(t_{\lfloor a i\rfloor}\right) f\left(t_{i}-\frac{t_{\lfloor a i\rfloor}}{a}\right) \\
& +\frac{a t_{i}-t_{\lfloor a i\rfloor}}{2 a}\left(\tilde{M}_{4}\left(t_{\lfloor a i\rfloor}\right) f\left(t_{i}-\frac{t_{\lfloor a i\rfloor}}{a}\right)\right)
\end{aligned}
$$

respectively, where $M_{1}\left(t_{i}\right)$ and $M_{2}\left(t_{i}\right)$ can be approximately calculated with the help of the formula given in (11) as

$$
\begin{aligned}
\tilde{M}_{1}\left(t_{i}\right) & =F\left(t_{i}\right)+\frac{h}{a} \sum_{j=1}^{\lfloor a i\rfloor-1} \tilde{M}_{1}\left(t_{j}\right) f\left(t_{i}-\frac{t_{j}}{a}\right) \\
& +\frac{h}{2 a} \tilde{M}_{1}\left(t_{\lfloor a i\rfloor}\right) f\left(t_{i}-\frac{t_{\lfloor a i\rfloor}}{a}\right) \\
& +\frac{a t_{i}-t_{\lfloor a i\rfloor}}{2 a}\left(\tilde{M}_{1}\left(t_{\lfloor a i\rfloor}\right) f\left(t_{i}-\frac{t_{\lfloor a i\rfloor}}{a}\right)\right)
\end{aligned}
$$

and

$$
\begin{aligned}
\tilde{M}_{2}\left(t_{i}\right) & =2 \tilde{M}_{1}\left(t_{i}\right)-F\left(t_{i}\right) \\
& +\frac{h}{a} \sum_{j=1}^{\lfloor a i\rfloor-1} \tilde{M}_{2}\left(t_{j}\right) f\left(t_{i}-\frac{t_{j}}{a}\right) \\
& +\frac{h}{2 a} \tilde{M}_{2}\left(t_{\lfloor a i\rfloor}\right) f\left(t_{i}-\frac{t_{\lfloor a i\rfloor}}{a}\right) \\
& +\frac{a t_{i}-t_{\lfloor a i\rfloor}}{2 a}\left(\tilde{M}_{2}\left(t_{\lfloor a i\rfloor}\right) f\left(t_{i}-\frac{t_{\lfloor a i\rfloor}}{a}\right)\right) .
\end{aligned}
$$

Note that these formulas in (12) and (13) are given previously by [17] and [15], respectively.

\section{ILLUSTRATIVE EXAMPLES}

In this section, we consider gamma, Weibull and lognormal distributions for the first interarrival time of the GP to illustrate the numerical method developed in previous section. As indicated in [10], the ratio parameter $a$ satisfies the condition $0.95 \leq a \leq 1.05$ for many real data sets fitted by the GP. Further, in the applications of the GP, we mostly encounter with values of $a$ which is less than 1. For this reason, the ratio parameter of the GP is taken as $a=0.95$ in each example. It is worth to 
noting that similar results are obtained for the different values of $a$. The value of $T$ is chosen to be at least $10 E\left(X_{1}\right)$.

In each example, we calculate the approximate values of the skewness and kurtosis of the GP model as

$$
\tilde{S}(t)=\frac{\tilde{M}_{3}(t)-3 \tilde{M}_{2}(t) \tilde{M}_{1}(t)+2 \tilde{M}_{1}^{3}(t)}{\left(\tilde{M}_{2}(t)-\tilde{M}_{1}^{2}(t)\right)^{3 / 2}}, t \geq 0
$$

and

$$
\tilde{K}(t)=\frac{\tilde{M}_{4}(t)-4 \tilde{M}_{3}(t) \tilde{M}_{1}(t)+6 \tilde{M}_{2}(t) \tilde{M}_{1}^{2}(t)-3 \tilde{M}_{1}^{4}(t)}{\left(\tilde{M}_{2}(t)-\tilde{M}_{1}^{2}(t)\right)^{2}}, t \geq 0
$$

respectively.

\section{Example 1. (Gamma distribution)}

Let $\{N(t), t \geq 0\}$ be a GP with ratio parameter $a=0.95$ and assume that the first interarrival time $X_{1}$ follows gamma distribution $\Gamma(2,1)$. Since $T=10 E\left(X_{1}\right)=$ 20 , we divide the interval $[0,20]$ into $n=2000$ subintervals with the equal width $h=$ 0.01 . The following Table 1 presents the approximate values of $M_{3}(t), M_{4}(t), S(t)$ and $K(t)$.

TABLE 1. Results for $X_{1} \sim \Gamma(2,1)$

\begin{tabular}{|c|c|c|c|c|}
\hline$t$ & $\tilde{M}_{3}(t)$ & $\tilde{M}_{4}(t)$ & $\tilde{S}(t)$ & $\tilde{K}(t)$ \\
\hline 0.1 & 0.0047 & 0.0047 & 14.5437 & 213.1503 \\
\hline 0.5 & 0.1016 & 0.1148 & 3.0185 & 10.9469 \\
\hline 1 & 0.3954 & 0.5569 & 1.4545 & 4.2591 \\
\hline 3 & 4.7898 & 11.9093 & 0.4792 & 3.1260 \\
\hline 5 & 17.2850 & 58.7025 & 0.3523 & 3.0903 \\
\hline 8 & 57.5463 & 267.5775 & 0.2646 & 3.0412 \\
\hline 10 & 101.6607 & 552.6060 & 0.2279 & 3.0256 \\
\hline 15 & 280.9871 & 2041.6477 & 0.1696 & 3.0086 \\
\hline 20 & 565.2640 & 5051.7662 & 0.1350 & 3.0045 \\
\hline
\end{tabular}




\section{Example 2. (Weibull distribution)}

Let $\{N(t), t \geq 0\}$ be a GP with ratio parameter $a=0.95$ and assume that the first interarrival time $X_{1}$ has Weibull distribution $\mathrm{W}(2,1)$. Since $T=10>$ $10 E\left(X_{1}\right)=5 \sqrt{\pi}$, the interval $[0,10]$ is divided into $n=1000$ subintervals with the equal width $h=0.01$. Thus, the approximate values of $M_{3}(t), M_{4}(t), S(t)$ and $K(t)$ are given in the Table 2 below.

TABLE 2. Results for $X_{1} \sim \mathrm{W}(2,1)$.

\begin{tabular}{|c|c|c|c|c|}
\hline$t$ & $\tilde{M}_{3}(t)$ & $\tilde{M}_{4}(t)$ & $\tilde{S}(t)$ & $\tilde{K}(t)$ \\
\hline 0.1 & 0.0101 & 0.0102 & 9.9125 & 99.8624 \\
\hline 0.5 & 0.2833 & 0.3573 & 1.5956 & 4.4056 \\
\hline 1 & 1.4831 & 2.6215 & 0.4613 & 2.9561 \\
\hline 2 & 9.6617 & 26.4035 & 0.4614 & 3.2020 \\
\hline 3 & 29.7577 & 108.7189 & 0.3757 & 3.1660 \\
\hline 4 & 65.4750 & 297.7482 & 0.3335 & 3.1183 \\
\hline 5 & 119.3496 & 645.6989 & 0.3003 & 3.0952 \\
\hline 8 & 403.4629 & 3151.2045 & 0.2350 & 3.0699 \\
\hline 10 & 699.8581 & 6486.4930 & 0.2059 & 3.0786 \\
\hline
\end{tabular}

\section{Example 3. (Lognormal distribution)}

Let $\{N(t), t \geq 0\}$ be a GP with ratio parameter $a=0.95$ and assume that the first interarrival time $X_{1}$ has lognormal distribution $\operatorname{LN}(0,1)$. Taking $T=$ $18>10 E\left(X_{1}\right)=10 e^{1 / 2}$, the interval $[0,18]$ is divided into $n=1800$ subintervals with the equal width $h=0.01$. Thus, we obtain the approximate values of $M_{3}(t)$, $M_{4}(t), S(t)$ and $K(t)$ in the Table 3 below.

TABle 3 . Results for $X_{1} \sim \operatorname{LN}(0,1)$.

\begin{tabular}{|c|c|c|c|c|}
\hline$t$ & $\tilde{M}_{3}(t)$ & $\tilde{M}_{4}(t)$ & $\tilde{S}(t)$ & $\tilde{K}(t)$ \\
\hline 0.1 & 0.0106 & 0.0107 & 9.5450 & 92.2791 \\
\hline 0.5 & 0.3473 & 0.4711 & 1.5313 & 4.3893 \\
\hline 1 & 1.4488 & 2.8111 & 0.8825 & 3.2820 \\
\hline 3 & 16.3445 & 58.9722 & 0.4168 & 2.8515 \\
\hline 5 & 52.0086 & 255.8734 & 0.2412 & 2.7747 \\
\hline 8 & 151.0759 & 997.1935 & 0.0829 & 2.7883 \\
\hline 10 & 249.7131 & 1898.0273 & 0.0096 & 2.8269 \\
\hline 15 & 613.5389 & 6027.1855 & -0.1164 & 2.9489 \\
\hline 18 & 911.0975 & 10036.7429 & -0.1682 & 3.0225 \\
\hline
\end{tabular}


It can be concluded from Tables 1-3 that the shape of the distribution of the GP converges to the normal distribution when the value of $t$ gets closer to the mean of the first interarrival time.

\section{CONCLUSIONS}

Integral equations satisfied by the third and fourth moment functions of a GP are derived. Further, we present an integral equation for the kth moment function as a generalization of the lower-order moment functions of the GP. In general manner, a numerical method established for solving the integral equation given for the kth moment function is presented. Then, we reduce this general structure to the solutions of the third and fourth integral equations to obtain their solutions. By using the solutions of the integral equation (3) and (4), skewness and kurtosis of the GP model are calculated for some lifetime distributions. According to the numerical calculations, as $t$ gets closer to $E\left(X_{1}\right)$, the shape of the distribution of the GP converges to the normal distribution. Note that more precise results can be obtained by taking smaller step width in numerical calculation of the integral equations since the accuracy of the approximation depends on the step width.

Authors Contributions Statement The authors jointly worked on the results and they read and approved the final version of the manuscript.

Declaration of Competing Interests The authors declare that they have no competing interest.

\section{REFERENCES}

[1] Arnold, R., Chukova, S., Hayakawa, Y., Marshall, S., Geometric-like processes: An overview and some reliability applications, Reliability Engineering and System Safety, 201 (2020). https://doi.org/10.1016/j.ress.2020.106990.

[2] Aydoğdu, H., Altında $\breve{g}$, Ö., Computation of the mean value and variance functions in geometric process, Journal of Statistical Computation and Simulation, 86:5 (2015), 986-995. https://doi.org/10.1080/00949655.2015.1047778.

[3] Aydoğdu, H., Karabulut, İ., Power series expansions for the distribution and mean value function of a geometric process with Weibull interarrival times, Naval Research Logistics, 61 (2014), 599-603. https://doi.org/10.1002/nav.21605.

[4] Aydoğdu, H., Karabulut, İ., Şen E., On the exact distribution and mean value function of a geometric process with exponential interarrival times, Statistics and Probability Letters, 83 (2013), 2577-2582. https://doi.org/10.1016/j.spl.2013.08.003.

[5] Bai, J., Pham, H., Repair-limit risk-free warranty policies with imperfect repair, IEEE Transactions on Systems, Man, and Cybernetics, Part A: Systems and Humans, 35:6 (2005), 756772. https://doi.org/10.1109/TSMCA.2005.851343.

[6] Braun, W. J., Li, W., Zhao, Y. Q., Properties of the geometric and related processes, Naval Research Logistics, 52, (2005), 607-616, https://doi.org/10.1002/nav.20099.

[7] Chan, J. S., Choy, S. B., Lam, C. P., Modeling electricity price using a threshold conditional autoregressive geometric process jump model, Communications in Statistics-Theory and Methods, 43 10-12 (2014), 2505-2515. https://doi.org/10.1080/03610926.2013.788714. 
[8] Chan, J. S., Yu, P. L., Lam, Y., Ho, A. P., Modelling SARS data using threshold geometric process. Statistics in Medicine, 25:11 (2006), 1826-1839. https://doi.org/10.1002/sim.2376 .

[9] Lam, Y., Geometric processes and replacement problem, Acta Mathematicae Applicatae Sinica, 4 (1988), 366-377. https://doi.org/10.1007/BF02007241.

[10] Lam, Y., Zhu, L. X., Chan, J. S. K., Liu, Q., Analysis of data from a series of events by a geometric process model, Acta Mathematicae Applicatae Sinica, 20(2) (2004), 263-82. https://doi.org/10.1007/s10255-004-0167-x.

[11] Lam, Y., The Geometric Process and Its Applications, Singapore, Word Scientific, 2007.

[12] Park, M, Pham, H., Warranty cost analyses using quasi-renewal processes for multicomponent systems, IEEE Trans Syst Man Cybern, Part A: Systems and Humans, 40:6 (2010), 1329-1340. https://doi.org/10.1109/TSMCA.2010.2046728.

[13] Pekalp, M. H., Aydoğdu, H., Türkman, K. F., Discriminating between some lifetime distributions in geometric counting processes, Communications in Statistics, Simulation and Computation, (2019). https://doi.org/10.1080/03610918.2019.1657452.

[14] Pekalp, M. H., Aydoğdu, H., An asymptotic solution of the integral equation for the second moment function in geometric processes, Journal of Computational and Applied Mathematics, 353 (2019), 179-190. https://doi.org/10.1016/j.cam.2018.12.014.

[15] Pekalp, M. H., Aydoğdu, H., An integral equation for the second moment function of a geometric process and its numerical solution, Naval Research Logistics, 65(2) (2018), 176184. https://doi.org/10.1002/nav.21791.

[16] Pham H., Wang H., A quasi-renewal process for software reliability and testing costs, IEEE Trans Syst Man Cybern, Part A: Systems and Humans, 31:6 (2001), 623-631. https://doi.org/10.1109/3468.983418.

[17] Tang, Y., Lam, Y., Numerical solution to an integral equation in geometric process, Journal of Statistical Computation and Simulation, 77 (2007), 549-560. https://doi.org/10.1080/10629360600565343.

[18] Wang, H., Pham, H., A quasi-renewal process and its applications in imperfect maintenance, Internat J Systems Sci., 27 (1996), 1055-1062. https://doi.org/10.1080/00207729608929311.

[19] Wu, D., Peng, R., Wu, S., A review of the extensions of the geometric process, Applications and Challenges. Quality and Reliability Engineering International, 36 (2020), 436-446. https://doi.org/10.1002/qre.2587.

[20] Wu, S., Doubly geometric process and applications, Journal of the Operational Research Society, 69 (1) (2018), 66-67. https://doi.org/10.1057/s41274-017-0217-4.

[21] Wu, S., Scarf, P., Decline and repair, and covariate effects, European Journal of Operational Research, 244 (1) (2015), 219-226. https://doi.org/10.1016/j.ejor.2015.01.041.

[22] Wu, S., Wang, G., The semi-geometric process and some properties, IMA Journal of Management Mathematics, 29 (2018), 229-245. https://doi.org/10.1093/imaman/dpx002.

[23] Zhang, M., Xie, M., Gaudoin, O., A bivariate maintenance policy for multi-state repairable systems with monotone process, IEEE Transactions of Reliability, 62(4) (2013), 876-886. https://doi.org/10.1109/TR.2013.2285042. 